\title{
Assessment of Atmospheric Pressure Plasma Treatment for Implant Osseointegration
}

\author{
Natalie R. Danna, ${ }^{1}$ Bryan G. Beutel, ${ }^{1}$ Nick Tovar, ${ }^{1}$ Lukasz Witek, ${ }^{2}$ Charles Marin, ${ }^{3}$ \\ Estevam A. Bonfante, ${ }^{3}$ Rodrigo Granato, ${ }^{3}$ Marcelo Suzuki, ${ }^{4}$ and Paulo G. Coelho ${ }^{15,6}$ \\ ${ }^{1}$ Department of Biomaterials and Biomimetics, New York University, 345 East 24th Street, Room 804 S, New York, NY 10010, USA \\ ${ }^{2}$ School of Chemical Engineering, Oklahoma State University, 423 Engineering North, Stillwater, OK 74078, USA \\ ${ }^{3}$ Department of Prosthodontics, University of São Paulo Bauru College of Dentistry, Al Otávio Pinheiro Brisola 9-75, \\ 17012-901 Bauru, SP, Brazil \\ ${ }^{4}$ Department of Prosthodontics, Tufts University School of Dental Medicine, 1 Kneeland Street, Boston, MA 02111, USA \\ ${ }^{5}$ Department of Periodontology and Implant Dentistry, New York University College of Dentistry, New York, USA \\ ${ }^{6}$ Division of Engineering, New York University Abu Dhabi, Abu Dhabi, UAE
}

Correspondence should be addressed to Bryan G. Beutel; bryanbeutel@gmail.com

Received 25 September 2014; Revised 18 January 2015; Accepted 23 January 2015

Academic Editor: Rolando A. Gittens

Copyright (C) 2015 Natalie R. Danna et al. This is an open access article distributed under the Creative Commons Attribution License, which permits unrestricted use, distribution, and reproduction in any medium, provided the original work is properly cited.

This study assessed the osseointegrative effects of atmospheric pressure plasma (APP) surface treatment for implants in a canine model. Control surfaces were untreated textured titanium $(\mathrm{Ti})$ and calcium phosphate $(\mathrm{CaP})$. Experimental surfaces were their 80 second air-based APP-treated counterparts. Physicochemical characterization was performed to assess topography, surface energy, and chemical composition. One implant from each control and experimental group (four in total) was placed in one radius of each of the seven male beagles for three weeks, and one implant from each group was placed in the contralateral radius for six weeks. After sacrifice, bone-to-implant contact (BIC) and bone area fraction occupancy (BAFO) were assessed. X-ray photoelectron spectroscopy showed decreased surface levels of carbon and increased $\mathrm{Ti}$ and oxygen, and calcium and oxygen, posttreatment for $\mathrm{Ti}$ and CaP surfaces, respectively. There was a significant $(P<0.001)$ increase in BIC for APP-treated textured Ti surfaces at six weeks but not at three weeks or for CaP surfaces. There were no significant $(P=0.57)$ differences for BAFO between treated and untreated surfaces for either material at either time point. This suggests that air-based APP surface treatment may improve osseointegration of textured Ti surfaces but not CaP surfaces. Studies optimizing APP parameters and applications are warranted.

\section{Introduction}

Osseointegration, the direct structural assimilation of bone to an implant, is a topic of particular importance to orthopaedic surgeons. In arthroplasty (e.g., around the prosthesis) and in trauma (e.g., around screws), bony anchorage onto the implant surface can make the difference between success and failure of reconstructive surgery. Surface modifications may increase the osseointegrative properties of implants [1-3], and optimizing bony ingrowth has been the subject of extensive investigation literature for years [4-8].

Osseointegration is postulated to proceed by adsorption of proteins, which then recruit osteoprogenitor cells onto the implant surface $[6,9,10]$. Cellular adhesion may be enhanced by manipulation of the implant's surface properties (e.g., charge, texture, and polarity) to yield a hospitable microenvironment on the implant surface [11-13]. Chemical (e.g., oxidation [14] and plasma treatment [4, 15]) and topographical (e.g., particulate coating, pressure blasting, and chemical abrasives) modifications have all been investigated as potential modifications [16-18]. Additionally, surface energy, a measure of unbonded surface atoms, is often examined to ascertain a material's ability to facilitate osseointegration $[9,19]$. High surface energy states foster cellular adhesion [20]. Surface energy may be divided into nonpolar (disperse) components and polar components, 
which accounts for polar groups, electric charges, and free radicals, as well as the roughness of the surface [21].

The focus of this study is the utilization of plasma treatment on the implant's surface to increase osseointegration. Plasma treatment may be utilized as an agent to alter a surface's properties in a variety of ways. Plasma may be generated with heat, termed thermal plasma treatment, often at low pressures [4]. Understandably, this presents environmental challenges such as achieving appropriate temperature and pressure for processing, limiting the utility of the procedure to industrial settings. Another technique for plasma generation occurs at ambient temperatures and atmospheric pressures, termed atmospheric pressure plasma (APP) treatment. In this process, argon gas has been described as an energy carrier, promoting the formation of reactive compounds on the implant surface [4]. Prior studies have demonstrated enhanced osseointegration through argon-based APP treatment $[4,19,22,23]$. However, investigations concerning the effect of other gases, especially compressed air that is readily available in operatories, are warranted if the ultimate goal is large-scale utilization of APP for increasing the osseointegration of implantable devices.

In the present study, we investigated the utilization of compressed air as an alternative to argon. As a safe, portable, and cost-effective technology, air-based APP treatment is thought to improve surface energy of implant surfaces by the removal of debris or molecules that may have become adsorbed during processing. Specifically, we evaluated airbased APP treatment on two widely used surfaces, namely, textured titanium ( $\mathrm{Ti}$, obtained by grit-blasting/acid-etching procedures) and calcium phosphate- (CaP-) coated implants, comparing treated to untreated surfaces in a canine radius model.

\section{Materials and Methods}

2.1. Physicochemical Evaluation. The textured Ti surfaces were obtained through grit-blasting/acid-etching (IntegraTi, Bicon LLC, Boston, MA) of plateau root form endosseous Ti-6Al-4V bulk alloy implants of $3.5 \mathrm{~mm}$ in diameter by $8 \mathrm{~mm}$ in length. CaP-coated (Integra-CP, Bicon LLC, Boston, MA) bulk alloy implants were also used in this study. The experimental set of implants was treated immediately prior to implantation with an APP application of compressed air for a total of 80 seconds $(20$ seconds per implant quadrant). The control groups were left untreated. Previous detailed physical/chemical characterization [24, 25] of these surfaces has shown that the CaP coatings were $\sim 20-30 \mu \mathrm{m}$ in thickness and presented $\sim 40 \%$ crystalline HA content. Surface roughness assessment has also shown that the plasma-sprayed hydroxyapatite surface roughness was higher $(1.8 \pm 0.25 \mu \mathrm{m})$ than the grit-blasted/acid-etched surfaces $(0.66 \pm 0.10 \mu \mathrm{m})[24,25]$.

The plasma was applied with a KinPen (INP, Greifswald, Germany) device (length $=190 \mathrm{~mm}$, diameter $=20 \mathrm{~mm}$, and weight $=170 \mathrm{~g}$ ). The KinPen was used for the generation of a plasma jet at atmospheric pressure connected to a highfrequency power supply $(1.5 \mathrm{MHz}, 2-6 \mathrm{kV}$ peak-to-peak, system power $230 \mathrm{~V}, 65 \mathrm{~W}$ ). The air supply was connected to a gas controller (Multi Gas Controller 647C, MKS Instruments, Andover, MA), which was set to flow at 5 standard liters per minute (l pm) [19]. The compressed air composition was the same as regular atmospheric composition at $16 \%$ oxygen, $1 \%$ hydrogen, and $78 \%$ nitrogen.

Physicochemical characterization was performed on nine implants from each group. The surface morphology was observed by scanning electron microscopy (SEM, Philips XL 30, Eindhoven, Netherlands) at 5000x magnification and an acceleration voltage of $15-20 \mathrm{kV}$ ( $n=3$ per group).

The surface energy was assessed using the Owens-WendtRabel-Kaelble method [26]. A micropipette (OCA 30, Data Physics Instruments GmbH, Filderstadt, Germany) was used to deposit $0.5 \mathrm{~mL}$ droplets of distilled water $\left(\mathrm{DI}-\mathrm{H}_{2} \mathrm{O}\right)$, ethylene glycol $\left(\mathrm{C}_{2} \mathrm{H}_{6} \mathrm{O}_{2}\right)$, and diiodomethane $\left(\mathrm{CH}_{2} \mathrm{I}_{2}\right)$ onto the surface of a member of each implant group. Software SCA30 (version 3.4.6, build 79) captured and analyzed each image. Each type droplet was applied on the apical flat surface of the plateau root form implants $(n=3)$.

Surface chemical characterization was performed by Xray photoelectron spectroscopy (XPS) at three different surface spots along the implant length. Three implants from each group were degassed to $10^{-7}$ torr and transferred under vacuum to a Kratos Axis 165 multitechnique XPS spectrometer (Kratos Analytical, Chestnut Ridge, NY). Survey and high-resolution spectra were obtained using a $165 \mathrm{~mm}$ mean radius concentric hemispherical analyzer operated at a constant pass energy of $160 \mathrm{eV}$ for survey and $80 \mathrm{eV}$ for highresolution scans (take-off angle of $90^{\circ}$, spot size of $150 \mu \mathrm{m} \times$ $150 \mu \mathrm{m})$.

2.2. In Vivo Model. For the in vivo study, seven adult male beagle dogs (number determined based on previous studies $[4,19,22,23])$, approximately 1.5 years of age, were used. The experimental protocol received the approval of the École Nationale Vétérinaire d'Alfort (Maisons-Alfort, Valde-Marne, France). NIH guidelines for the care and use of laboratory animals and policies of the Animal Welfare Act were observed. The beagles remained in the facility for an approximate two-week acclimation period prior to any surgical intervention.

All surgical procedures were performed under general anesthesia. Intramuscular (IM) atropine sulfate $(0.044 \mathrm{mg} / \mathrm{kg})$ and xylazine chlorate $(8 \mathrm{mg} / \mathrm{kg})$ were administered for preanesthesia. General anesthesia was then obtained following an IM injection of ketamine chlorate $(15 \mathrm{mg} / \mathrm{kg})$. Following skin preparation, a $5 \mathrm{~cm}$ incision was made and the deeper tissues were dissected to expose the diaphysis of the radius.

Four implants (untreated $\mathrm{Ti}$, APP-treated $\mathrm{Ti}$, untreated $\mathrm{CaP}$, and APP-treated $\mathrm{CaP}$ ) were placed along each radius. The implants were press fit into $3.5 \mathrm{~mm}$ drill holes made with a power drill (1200 rpm for the pilot hole, $800 \mathrm{rpm}$ for sequentially larger bits) under continuous saline irrigation. They were placed from proximal to distal directions, approximately $1 \mathrm{~cm}$ apart, in various positions in the sequence in order to eliminate any confounding of the results based on implant location in vivo. At the initial surgery, implants were placed only in the left limb to be analyzed six weeks 


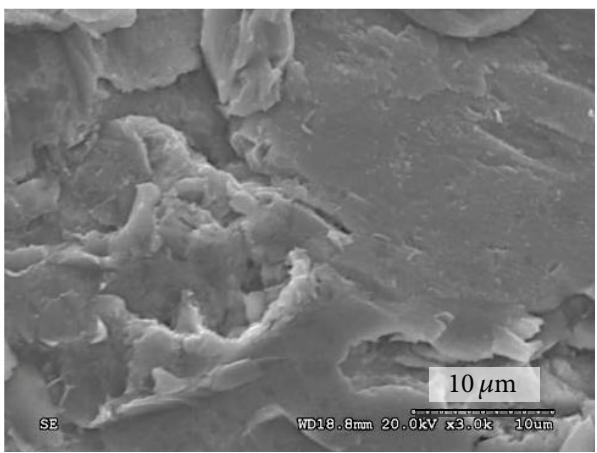

(a)

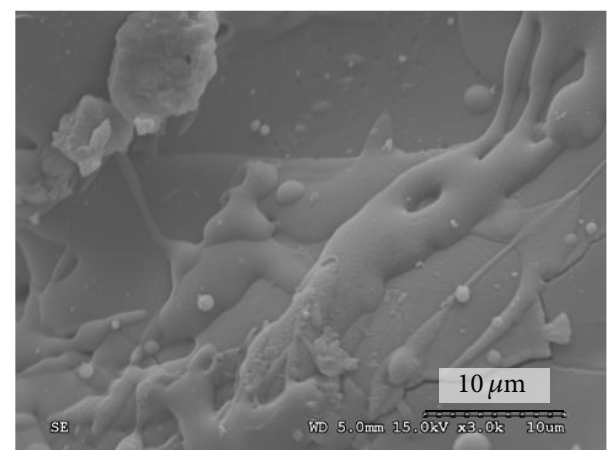

(b)

FIGURE 1: Scanning electron micrographs of (a) titanium (Ti) and (b) calcium phosphate (CaP) implant surfaces before atmospheric pressure plasma (APP) treatment.

postoperatively. Three weeks later, implants for a three-week postoperative assessment were placed in the right limb in a second procedure.

Closure was achieved with standard layered suturing techniques with VICRYL 4-0 (Ethicon Johnson, Miami, FL) for deep tissues and nylon 4-0 (Ethicon Johnson, Miami, FL) for skin. The dogs remained in the animal care facility and received antibiotics (benzyl penicillin benzathine $20.00 \mathrm{IU} / \mathrm{kg}$ ) and anti-inflammatory medication (ketoprofen $1 \%, 1 \mathrm{~mL} / 5 \mathrm{~kg}$ ) for pain control. Euthanasia was carried out by anesthesia overdose six weeks after the first surgical procedure.

2.3. Histomorphologic Evaluation. At necropsy, the radii with implants were retrieved by sharp dissection. The bone blocks were kept in $10 \%$ buffered formalin solution for 24 hours, washed in running water for 24 hours, and gradually dehydrated in a series of alcohol solutions ranging from 70 to $100 \%$ ethanol. Following dehydration, the samples were embedded in a methacrylate-based resin (Technovit 9100, Heraeus Kulzer $\mathrm{GmbH}$, Wehrheim, Germany) according to the manufacturer's instructions. The blocks were then cut into slices ( $300 \mu \mathrm{m}$ thick) centering the implant along its long axis with a precision diamond saw (Isomet 2000, Buehler Ltd., Lake Bluff, IL) and glued to acrylic plates with an acrylate-based cement (Technovit 7210 VLC, Heraeus Kulzer $\mathrm{GmbH}$, Wehrheim, Germany), and a 24-hour setting time was allowed prior to grinding and polishing. The sections were then reduced to a final thickness of $\sim 30 \mu \mathrm{m}$ by means of a series of silicon carbide ( $\mathrm{SiC})$ abrasive papers (280, 400, 800, 1200, and 2500; Buehler Ltd., Lake Bluff, IL) in a grinding/polishing machine (Metaserv 3000, Buehler Ltd., Lake Bluff, USA) under water irrigation. The sections were then stained with toluidine blue and subjected to optical microscopy for histomorphologic evaluation.

The histologic features were evaluated at 50x-200x magnification (Leica DM2500M, Leica Microsystems GmbH, Wetzlar, Germany). The bone-to-implant contact (BIC) was determined by computer software (Leica Application Suite, Leica Microsystems GmbH, Wetzlar, Germany). The length of bone along the implant perimeter was measured and divided by e total implant perimeter to calculate the BIC percentage. The bone area fraction occupancy (BAFO) between plateaus was determined at 100x magnification with the same microscope and software by subtracting the percentage area occupied by bone from the total available area within the healing chambers.

2.4. Statistics. Statistical analysis was performed with SPSS (version 19, New York, NY). XPS data were evaluated by oneway ANOVA at 95\% level of significance. For all outcomes concerning the animal study, statistical significance was set to a $95 \%$ level of confidence and the number of dogs was considered the statistical unit for all comparisons. For the histomorphometric-dependent variables BIC and BAFO, a GLM ANOVA (general linear model) was employed including surface group and time in vivo as independent variables (surgical site position was preliminarily evaluated and, due to a lack of effect on BIC and BAFO, was ultimately excluded from further analysis).

\section{Results}

3.1. Surface Characterization. The SEM micrographs of the implant surfaces revealed a grit-blasted textured surface (Figure 1(a)) on the Ti implant and a textured microstructure surface on the CaP implant (Figure 1(b)). The surface energy assessment showed a substantial increase of approximately $15 \mathrm{mN} / \mathrm{m}$ in the polar component and a small increase $(5 \mathrm{mN} / \mathrm{m})$ of the disperse component of the Ti implant group immediately after plasma treatment (Figure 2). Both the polar and disperse components of the CaP implant group showed increases $(9 \mathrm{mN} / \mathrm{m}$ and $5 \mathrm{mN} / \mathrm{m}$, resp.) following the plasma application.

The XPS survey analysis of the control and air APPtreated implants showed peaks of $\mathrm{Ti}$ and $\mathrm{O}$ for both treated and untreated Ti surfaces. High-resolution spectrum evaluation demonstrated that for both surfaces carbon (C) was observed primarily as hydrocarbon $(\mathrm{C}-\mathrm{C}, \mathrm{C}-\mathrm{H})$ with lower levels of oxidized $\mathrm{C}$ forms. For the untreated Ti surfaces, XPS detected the atomic percent values (mean \pm SD) of $45.0 \pm 5.1$ for $\mathrm{C}, 1.5 \pm 0.5$ for aluminum $(\mathrm{Al}), 0.1 \pm 0.2$ for nitrogen $(\mathrm{N})$, $14 \pm 4.7$ for $\mathrm{Ti}$, and $37.0 \pm 3.6$ for $\mathrm{O}$ (Table 1 ). Vanadium (V) was detected at trace levels. Compared to the control group, the air APP-treated Ti surfaces showed decreased levels of 


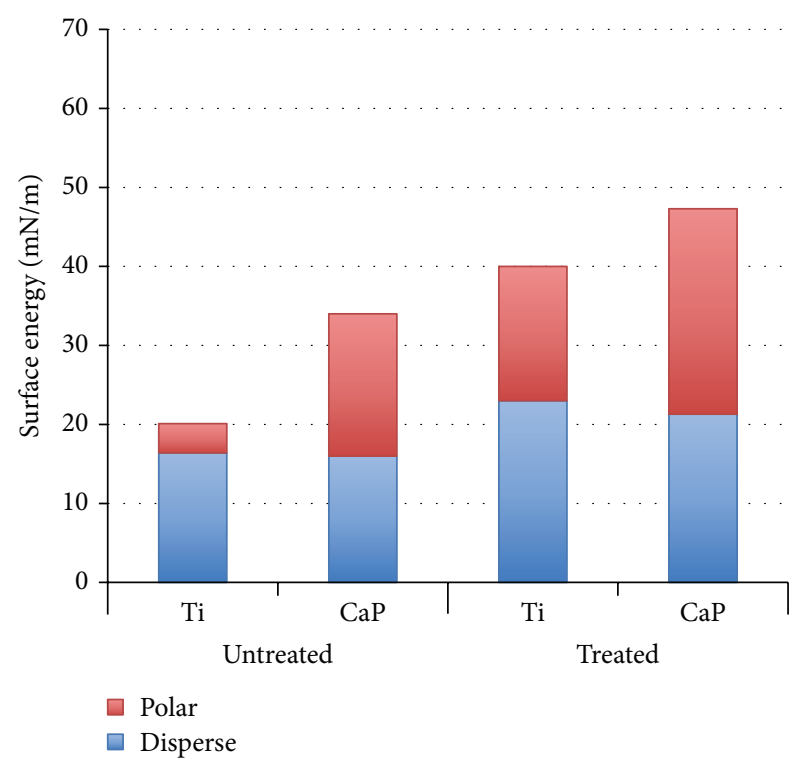

FIGURE 2: Surface energy bar graph for polar and disperse components of the titanium $(\mathrm{Ti})$ and calcium phosphate $(\mathrm{CaP})$ groups before (untreated) and after (treated) atmospheric pressure plasma treatment.

TABLE 1: X-ray photoelectron spectroscopy spectra for titanium (Ti) and calcium phosphate $(\mathrm{CaP})$, both untreated and atmospheric pressure plasma- (APP-) treated. Mean (SD) values are provided.

\begin{tabular}{lcccc}
\hline Chemical & \multicolumn{2}{c}{ Ti surface } & \multicolumn{2}{c}{ CaP surface } \\
element (\%) & Untreated & APP-treated & Untreated & APP-treated \\
\hline Al2p & $1.5(0.5)$ & $* *$ & $* *$ & $* *$ \\
C1s & $45.0(5.1)$ & $38.0(4.7)$ & $38.0(4.2)$ & $22.0(4.5)$ \\
N1s & $0.1(0.2)$ & - & - & - \\
Ca2p & - & - & $11.0(2.5)$ & $12.0(2.3)$ \\
O1s & $37.6(3.6)$ & $43.0(4.3)$ & $42.0(5.2)$ & $48.0(3.1)$ \\
P2p & - & - & $7.0(1.3)$ & $10.0(2.4)$ \\
Ti2p & $14.0(4.7)$ & $19.0(3.9)$ & $* *$ & $* *$ \\
V2p3 & $* *$ & $* *$ & $* *$ & $* *$ \\
\hline
\end{tabular}

Al: aluminum; C: carbon; N: nitrogen; Ca: calcium; O: oxygen; P: phosphate; $\mathrm{V}$ : vanadium.

${ }^{* *}$ Only trace amounts were present.

$\mathrm{C}(38.0 \pm 4.7)$ and increased levels of $\mathrm{Ti}(19.0 \pm 3.9)$ and $\mathrm{O}$ $(43.0 \pm 4.3)$. Similar values were observed for $\mathrm{Al}, \mathrm{N}$, and $\mathrm{V}$ in the control and experimental groups.

For the CaP surfaces, the XPS survey analysis of the implant surface showed peaks of calcium (Ca), C, O, and phosphate $(\mathrm{P})$ for both the control and air APP-treated samples. High-resolution spectrum evaluation revealed that for both surfaces $\mathrm{C}$ was observed primarily as hydrocarbon with lower levels of oxidized $\mathrm{C}$ forms. For both the treated and untreated groups, $\mathrm{Ca}$ and $\mathrm{P}$ were detected in varied atomic concentrations. For the $\mathrm{CaP}$ group, the atomic percent values $($ mean $\pm \mathrm{SD}$ ) were $38.0 \pm 4.2$ for $\mathrm{C}, 42.0 \pm 5.2$ for $\mathrm{O}, 11.0 \pm 2.5$ for $\mathrm{Ca}$, and $7.0 \pm 1.3$ for $\mathrm{P}$ (Table 1). When compared to the control CaP group, the APP-treated CaP implants presented increases in $\mathrm{O}(48.0 \pm 3.1), \mathrm{Ca}(12.0 \pm 2.3)$, and $\mathrm{P}(10.0 \pm 2.4)$ atomic percent levels. A decrease in $\mathrm{C}$ content was observed at $22.0 \pm 4.5$ atomic percent.

3.2. In Vivo Model. The animal surgical procedures and follow-up demonstrated no complications or other clinical concerns. Therefore, no implant was excluded due to clinical instability, which was clinically determined after euthanization.

The BIC results as a function of time in vivo and implant surface presented significant differences (both $P<0.001$ ) (Figure 3). No difference between the treated and untreated groups of either the $\mathrm{CaP}$ or Ti surface was observed at three weeks (Figure 4$)$. A significant $(P<0.001)$ difference was observed in BIC for the Ti implant but not for the CaP one at six weeks. BAFO measurements did not show a significant $(P=0.57)$ difference between the treated and untreated groups (Figure 3). However, there was a significant difference in BAFO between 3 and 6 weeks in vivo $(P<0.001)$ (Figure 3). No differences in BAFO were noted between any groups at either three or six weeks in vivo (Figure 5).

Qualitative evaluation of the stained histological sections of the untreated groups after three weeks in vivo demonstrated minimal woven bone in proximity to the implant surface (Figures 6(a) and 6(b)). Their APP-treated counterparts, however, presented a more well-distributed bone formation within the plateau and a higher degree of bone formation in proximity to the implant surface (Figures 6(c) and 6(d)). At six weeks, the untreated surfaces presented lower degrees of bone formation in proximity to the implant surface (Figures $6(\mathrm{e})$ and $6(\mathrm{~F})$ ) relative to their APP-treated counterparts (Figures $6(\mathrm{~g})$ and $6(\mathrm{~h})$ ). Such differences were more pronounced between the untreated and the APP-treated Ti surfaces. An overall large increase in bone formation occurred for all groups from 3 to 6 weeks.

\section{Discussion}

Endosseous implant surfaces have evolved from presenting the as-machined turned surface towards textured Ti surfaces (obtained by additive or subtractive engineering methods) under the unequivocal support that osseoconduction of textured surfaces is substantially improved relative to smoother surfaces $[5,11]$. Recent research has also convincingly demonstrated that calcium- and phosphate-based coatings on $\mathrm{Ti}$ surfaces further increase the osseoconductivity obtained through texturing Ti surfaces alone [5, 11]. A plethora of in vivo laboratory models, including both surfaces evaluated in the present study, have shown that, regardless of the animal model, the plasma-sprayed $\mathrm{CaP}$ surface presents higher degrees of measurable osseointegration parameters and biomechanical fixation at early implantation times in vivo [27-29].

While the plasma spraying of $\mathrm{CaP}$ coatings is performed under controlled atmosphere and high temperatures in order to coat Ti implants with a $20-50 \mu \mathrm{m}$ thickness and is thus an industrial process that requires safety measures $[5,11]$, the APP technology evaluated in the present study 


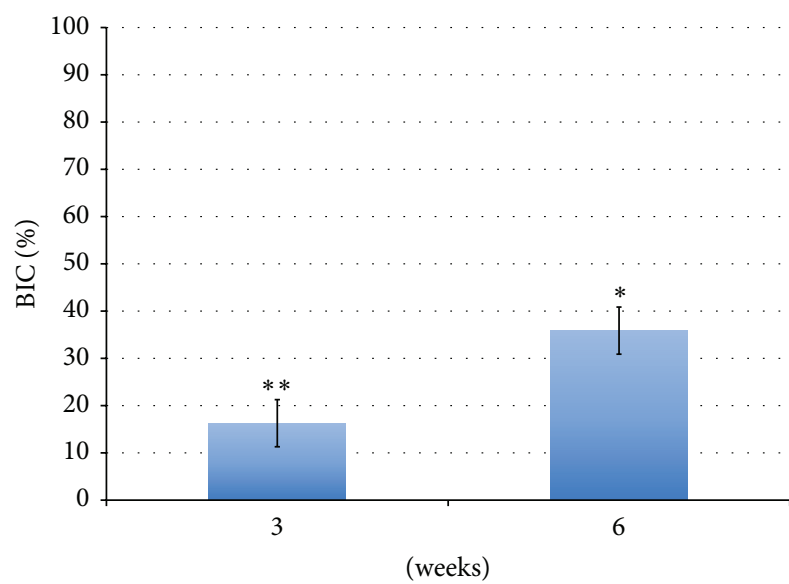

(a)

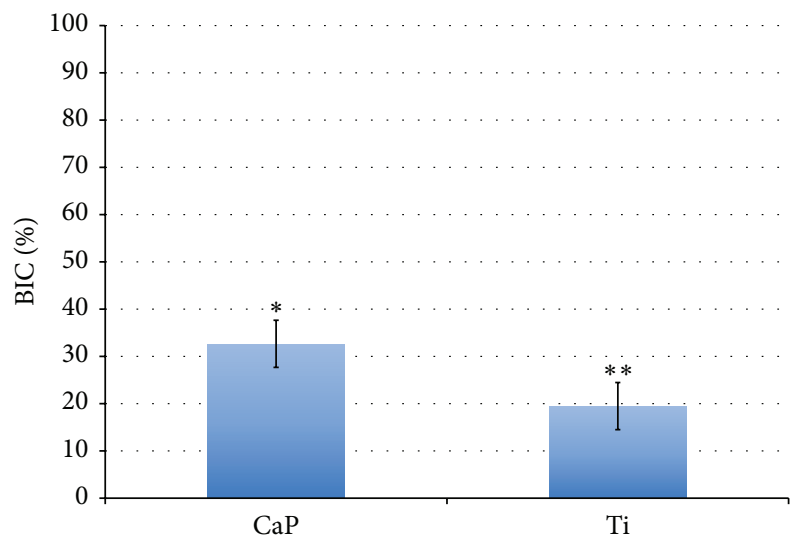

(c)

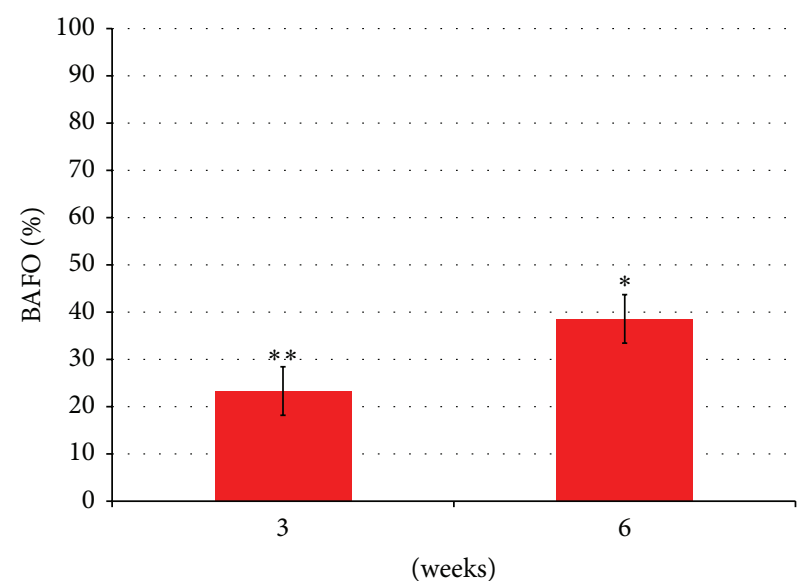

(b)

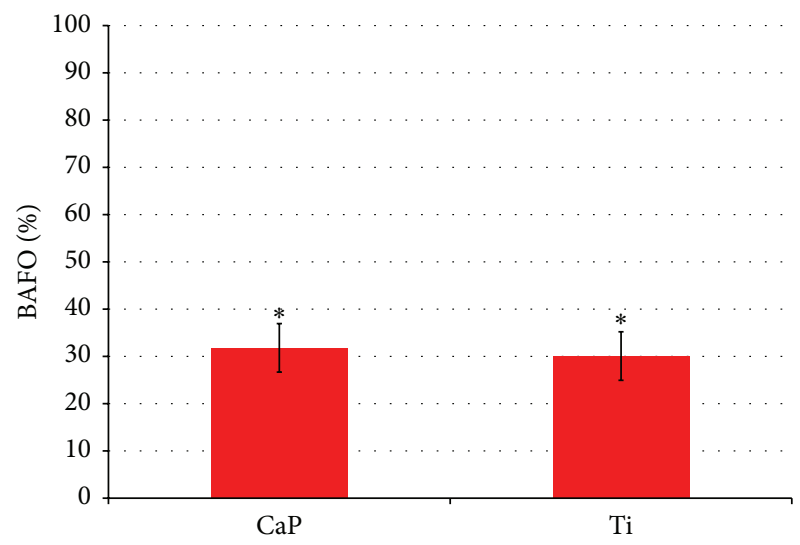

(d)

FIGURE 3: In vivo bone and implant characteristics as a function of ((a) and (b)) time in vivo collapsed over surface type and ((c) and (d)) implant surface type collapsed over time in vivo. BIC: bone-to-implant contact; BAFO: bone area fraction occupancy; Ti: titanium; and CaP: calcium phosphate. Asterisks indicate statistically homogeneous groups (different numbers of asterisks depict that groups were statistically different at $P<0.05$ ) assessed for $n=7$ animals.

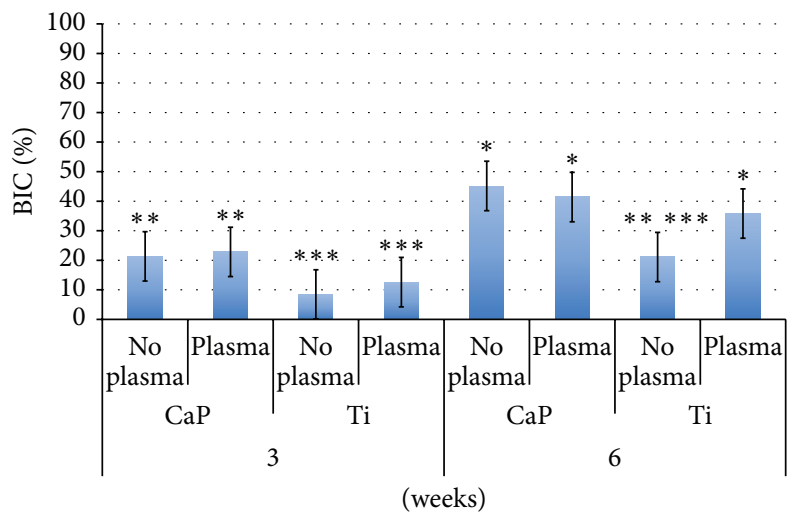

FIGURE 4: Osseointegration of implants by bone-to-implant contact (BIC). Mean values with standard deviation bars are provided for the untreated (no plasma) and atmospheric pressure plasma-treated (plasma) titanium (Ti) and calcium phosphate (CaP) groups at respective experimental time periods. Asterisks indicate statistically homogeneous groups (different numbers of asterisks depict that groups were statistically different at $P<0.05$ ) assessed for $n=7$ animals. 


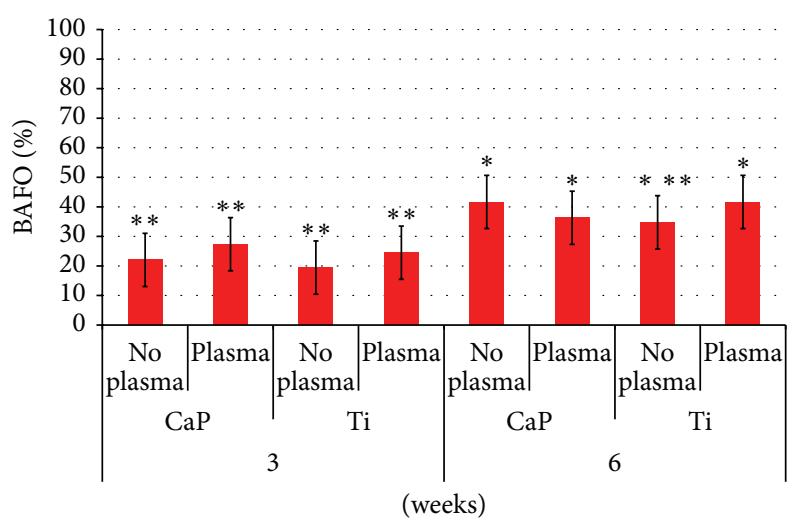

FIGURE 5: Osseointegration of implants by bone area fraction occupancy (BAFO). Mean values with standard deviation bars are provided for the untreated (no plasma) and atmospheric pressure plasma-treated (plasma) titanium (Ti) and calcium phosphate (CaP) groups at respective experimental time periods. Asterisks indicate statistically homogeneous groups (different numbers of asterisks depict that groups were statistically different at $P<0.05$ ) assessed for $n=7$ animals.
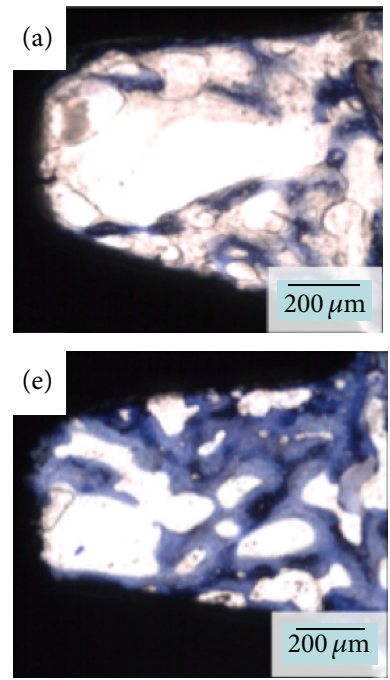
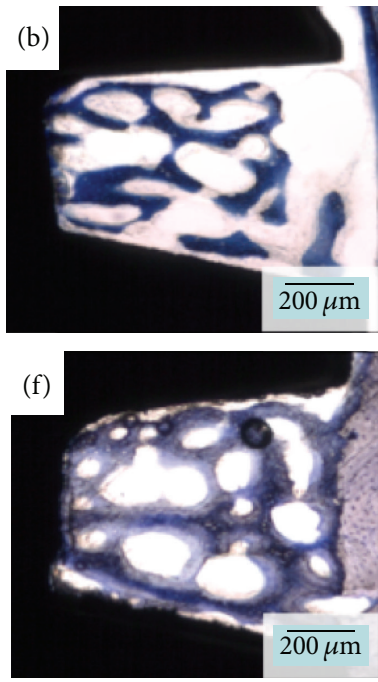
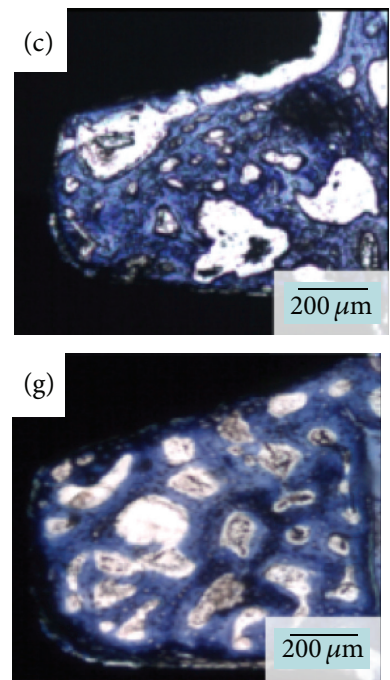
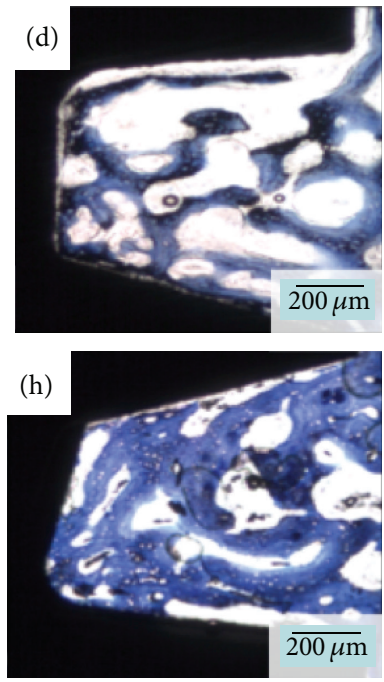

FIGURE 6: Representative overview of the histological micrographs. Untreated calcium phosphate (CaP) implants at three (a) and six (e) weeks, respectively; untreated titanium (Ti) implant at three (b) and six (f) weeks, respectively; atmospheric pressure plasma- (APP-) treated $\mathrm{CaP}$ implants at three (c) and six (g) weeks; and APP-treated Ti group at three (d) and six (h) weeks.

has been previously utilized to surface treat implants of varied compositions and textures immediately prior to their implantation $[4,19,22,23]$. APP usually operates at room temperature, substantially lower temperatures compared to the coating processing of hot plasma (at several thousand degrees). Thus, APP's ability to deliver surface modification [30] at room temperature through portable equipment is a promising technology for facilitating early osseointegration of any biocompatible implant surface regardless of its chemistry and topography. The characteristics of the technology make it more affordable and conducive to use in the operating room immediately prior to implant placement $[4,19,22,23]$. It must be noted that other methods have been attempted to decrease surface contamination while increasing its energy. For instance, Buser et al. [31] have shown that increased levels of osseointegration can be obtained through an industrial surface cleaning and packaging method in a liquid phase relative to controls. Different from the proprietary process described by Buser et al. [31], a photofunctionalization method that can be utilized in the operating room immediately prior to implantation has been extensively described by Ogawa et al. [32-34] at both in vitro and in vivo settings. Both methods have demonstrated efficiency in increasing osseointegration levels.

Previous studies have used the KinPen device supplied with argon gas and indicated that the treatment enhanced osseointegration at early time points in vivo $[4,19,22$, 23]. Of these, some have assessed the same $\mathrm{Ti}$ and $\mathrm{CaP}$ surfaces utilized in the present study, and while over a $300 \%$ increase in osseointegration was obtained for argon-based APP-treated Ti surfaces relative to controls at three weeks in vivo [4], a much smaller size effect of approximately $80 \%$ was observed for the argon-based APP-treated CaP surfaces relative to their untreated counterparts at the same implantation 
time [22]. From both studies regarding physicochemical characterization, the increase in surface energy was more remarkable for the Ti surfaces compared to the $\mathrm{CaP}$ surfaces, and such relative differences between argon APP-treated and untreated surfaces are thought to be the reason for the relative differences in osseointegration.

The present study investigated whether exchanging argon for compressed air in the APP application furnished the same osseointegrative benefits. Relative to previous studies, both physicochemical and in vivo results depicted that compressed air APP treatment did increase surface energy and facilitated earlier osseointegration relative to controls, but such increases were not as remarkable as the results obtained with argon gas $[4,22]$. The surface energy and XPS results showed that surface elemental chemistry was modified by the air-based APP treatment and that this change resulted in a higher degree of exposure of the surface chemical elements, mainly at the expense of the removal of adsorbed $\mathrm{C}$ species immediately after plasma treatment [35]. For both Ti and CaP surfaces, increases were noted but were not nearly as high as previously reported for argon gas $[4,22]$. A limitation of the current investigation along with previous work $[4,22]$ is that no assessment of surface texture modification at the micrometer and nanometer scale was made after the different APP regimens were performed, thereby warranting further assessment of this important surface parameter.

The histological evaluation revealed that the interaction between tissue and implant surface occurred at three weeks in vivo for both experimental groups (APP-treated $\mathrm{CaP}$ and APP-treated $\mathrm{Ti}$ ), while the untreated $\mathrm{CaP}$ and $\mathrm{Ti}$ groups displayed lower degrees of bone formation in proximity to the implant surface interaction until six weeks in vivo. Overall, the CaP implant surface did not benefit from the APP treatment, while increased bone-to-implant contact at 6 weeks was observed for the APP-treated Ti surface. Comparing the current study to previous studies $[4,22]$ of argon-based NTP treatment, the air-based plasma did not increase the surface energy to the same degree as argon. Additionally, the carbon present on the implant surface did not decrease to the same extent as it had with the argon APP treatment. Thus, given the previous and current results, it can be concluded that the air-based APP treatment is not as effective as its argon-based counterpart.

\section{Conclusions}

While air-based APP treatment did not surpass argon-based treatment, the results of this study do not invalidate its utility either. It would be of interest to investigate the technology's impact on bone formation when longer treatment times are employed. Additionally, assessment of the biomechanical integrity of the bone-implant construct would provide information about the quality of formed bone, which could be considered in addition to this study's quantitative assessment.

\section{Disclaimer}

The funders had no role in study design, data collection and analysis, decision to publish, or preparation of the paper.

\section{Conflict of Interests}

The authors declare that there is no conflict of interests regarding the publication of this paper.

\section{Acknowledgment}

This study was partially supported by Bicon LLC (Boston, MA).

\section{References}

[1] R. Branemark, L. Emanuelsson, A. Palmquist, and P. Thomsen, "Bone response to laser-induced micro- and nano-size titanium surface features," Nanomedicine: Nanotechnology, Biology, and Medicine, vol. 7, no. 2, pp. 220-227, 2011.

[2] P. G. Coelho and R. Jimbo, "Osseointegration of metallic devices: current trends based on implant hardware design," Archives of Biochemistry and Biophysics, vol. 561, pp. 99-108, 2014.

[3] P. G. Coelho, R. Jimbo, N. Tovar, and E. A. Bonfante, "Osseointegration: hierarchical designing encompassing the macrometer, micrometer, and nanometer length scales," Dental Materials, vol. 31, pp. 37-52, 2015.

[4] P. G. Coelho, G. Giro, H. S. Teixeira et al., "Argon-based atmospheric pressure plasma enhances early bone response to rough titanium surfaces," Journal of Biomedical Materials Research Part A, vol. 100, no. 7, pp. 1901-1906, 2012.

[5] P. G. Coelho, J. M. Granjeiro, G. E. Romanos et al., "Basic research methods and current trends of dental implant surfaces," Journal of Biomedical Materials Research Part B: Applied Biomaterials, vol. 88B, no. 2, pp. 579-596, 2009.

[6] T. Albrektsson and A. Wennerberg, "Oral implant surfaces: part 1-review focusing on topographic and chemical properties of different surfaces and in vivo responses to them," International Journal of Prosthodontics, vol. 17, no. 5, pp. 536-543, 2004.

[7] W.-D. Müeller, U. Gross, T. Fritz et al., "Evaluation of the interface between bone and titanium surfaces being blasted by aluminium oxide or bioceramic particles," Clinical Oral Implants Research, vol. 14, no. 3, pp. 349-356, 2003.

[8] C. M. Stanford, "Surface modifications of dental implants," Australian Dental Journal, vol. 53, supplement 1, pp. S26-S33, 2008.

[9] R. E. Baier, A. E. Meyer, J. R. Natiella, R. R. Natiella, and J. M. Carter, "Surface properties determine bioadhesive outcomes: methods and results," Journal of Biomedical Materials Research, vol. 18, no. 4, pp. 337-355, 1984.

[10] R. Jimbo, M. Ivarsson, A. Koskela, Y.-T. Sul, and C. B. Johansson, "Protein adsorption to surface chemistry and crystal structure modification of titanium surfaces," Journal of Oral and Maxillofacial Research, vol. 1, no. 3, p. e3, 2010.

[11] D. M. Dohan Ehrenfest, P. G. Coelho, B.-S. Kang, Y.-T. Sul, and T. Albrektsson, "Classification of osseointegrated implant surfaces: materials, chemistry and topography," Trends in Biotechnology, vol. 28, no. 4, pp. 198-206, 2010.

[12] T. Albrektsson and A. Wennerberg, "Oral implant surfaces: part 2-review focusing on clinical knowledge of different surfaces," International Journal of Prosthodontics, vol. 17, no. 5, pp. 544$564,2004$.

[13] T. Albrektsson, D. Buser, and L. Sennerby, "On crestal/marginal bone loss around dental implants," The International Journal of Oral \& Maxillofacial Implants, vol. 27, no. 4, pp. 736-738, 2012. 
[14] R. Jimbo, T. Sawase, K. Baba, T. Kurogi, Y. Shibata, and M. Atsuta, "Enhanced initial cell responses to chemically modified anodized titanium," Clinical Implant Dentistry and Related Research, vol. 10, no. 1, pp. 55-61, 2008.

[15] L. V. Carlsson, T. Alberktsson, and C. Berman, "Bone response to plasma-cleaned titanium implants," The International Journal of Oral \& Maxillofacial Implants, vol. 4, no. 3, pp. 199-204, 1989.

[16] R. Chowdhary, A. Halldin, R. Jimbo, and A. Wennerberg, "Influence of micro threads alteration on osseointegration and primary stability of implants: an FEA and in vivo analysis in rabbits," Clinical Implant Dentistry and Related Research, 2013.

[17] M. Hayashi, R. Jimbo, L. Lindh et al., "In vitro characterization and osteoblast responses to nanostructured photocatalytic $\mathrm{TiO}_{2}$ coated surfaces," Acta Biomaterialia, vol. 8, no. 6, pp. 2411-2416, 2012.

[18] J. Karlsson, R. Jimbo, H. M. Fathali et al., "In vivo biomechanical stability of osseointegrating mesoporous $\mathrm{TiO}_{2}$ implants," Acta Biomaterialia, vol. 8, no. 12, pp. 4438-4446, 2012.

[19] F. P. S. Guastaldi, D. Yoo, C. Marin et al., "Plasma treatment maintains surface energy of the implant surface and enhances osseointegration," International Journal of Biomaterials, vol. 2013, Article ID 354125, 6 pages, 2013.

[20] T. Miyauchi, M. Yamada, A. Yamamoto et al., "The enhanced characteristics of osteoblast adhesion to photofunctionalized nanoscale $\mathrm{TiO}_{2}$ layers on biomaterials surfaces," Biomaterials, vol. 31, no. 14, pp. 3827-3839, 2010.

[21] D. Staack, A. Fridman, A. Gutsol, Y. Gogotsi, and G. Friedman, "Nanoscale corona discharge in liquids, enabling nanosecond optical emission spectroscopy," Angewandte Chemie International Edition, vol. 47, no. 42, pp. 8020-8024, 2008.

[22] G. Giro, N. Tovar, L. Witek et al., "Osseointegration assessment of chairside argon-based nonthermal plasma-treated CaP coated dental implants," Journal of Biomedical Materials Research Part A, vol. 101, no. 1, pp. 98-103, 2013.

[23] H. S. Teixeira, C. Marin, L. Witek et al., "Assessment of a chairside argon-based non-thermal plasma treatment on the surface characteristics and integration of dental implants with textured surfaces," Journal of the Mechanical Behavior of Biomedical Materials, vol. 9, pp. 45-49, 2012.

[24] P. G. Coelho, G. Cardaropoli, M. Suzuki, and J. E. Lemons, "Histomorphometric evaluation of a nanothickness bioceramic deposition on endosseous implants: a study in dogs," Clinical Implant Dentistry and Related Research, vol. 11, no. 4, pp. 292302, 2009.

[25] P. G. Coelho and J. E. Lemons, "Physico/chemical characterization and in vivo evaluation of nanothickness bioceramic depositions on alumina-blasted/acid-etched Ti-6Al-4V implant surfaces," Journal of Biomedical Materials Research A, vol. 90, no. 2, pp. 351-361, 2009.

[26] R. J. Good and C. J. van Oss, Modern Approaches to Wettability: Theory and Applications, Plenum Press, New York, NY, USA, 1992.

[27] P. G. Coelho, C. Marin, R. Granato, G. Giro, M. Suzuki, and E. A. Bonfante, "Biomechanical and histologic evaluation of nonwashed resorbable blasting media and alumina-blasted/acidetched surfaces," Clinical Oral Implants Research, vol. 23, no. 1, pp. 132-135, 2012.

[28] M. Suzuki, M. D. Calasans-Maia, C. Marin et al., "Effect of surface modifications on early bone healing around plateau root form implants: an experimental study in rabbits," Journal of Oral and Maxillofacial Surgery, vol. 68, no. 7, pp. 1631-1638, 2010.
[29] D. Yoo, N. Tovar, R. Jimbo et al., "Increased osseointegration effect of bone morphogenetic protein 2 on dental implants: an in vivo study," Journal of Biomedical Materials Research A, vol. 102, no. 6, pp. 1921-1927, 2014.

[30] K. H. Becker, U. Kogelschatz, K. H. Schoenbach, and R. J. Barker, Non-Equilibrium Air Plasmas at Atmospheric Pressure, Taylor \& Francis, New York, NY, USA, 2004.

[31] D. Buser, N. Broggini, M. Wieland et al., "Enhanced bone apposition to a chemically modified SLA titanium surface," Journal of Dental Research, vol. 83, no. 7, pp. 529-533, 2004.

[32] M. Hirota, T. Ikeda, M. Tabuchi, T. Iwai, I. Tohnai, and T. Ogawa, "Effect of ultraviolet-mediated photofunctionalization for bone formation around medical titanium mesh," Journal of Oral and Maxillofacial Surgery, vol. 72, no. 9, pp. 1691-1702, 2014.

[33] T. Ogawa, "Ultraviolet photofunctionalization of titanium implants," The International Journal of Oral \& Maxillofacial Implants, vol. 29, no. 1, pp. e95-e102, 2014.

[34] S.-W. Pyo, Y. B. Park, H. S. Moon, J.-H. Lee, and T. Ogawa, "Photofunctionalization enhances bone-implant contact, dynamics of interfacial osteogenesis, marginal bone seal, and removal torque value of implants: a dog jawbone study," Implant Dentistry, vol. 22, no. 6, pp. 666-675, 2013.

[35] P. G. Coelho and J. E. Lemons, "Physico/chemical characterization and in vivo evaluation of nanothickness bioceramic depositions on alumina-blasted/acid-etched Ti-6Al-4V implant surfaces," Journal of Biomedical Materials Research Part A, vol. 90, no. 2, pp. 351-361, 2009. 

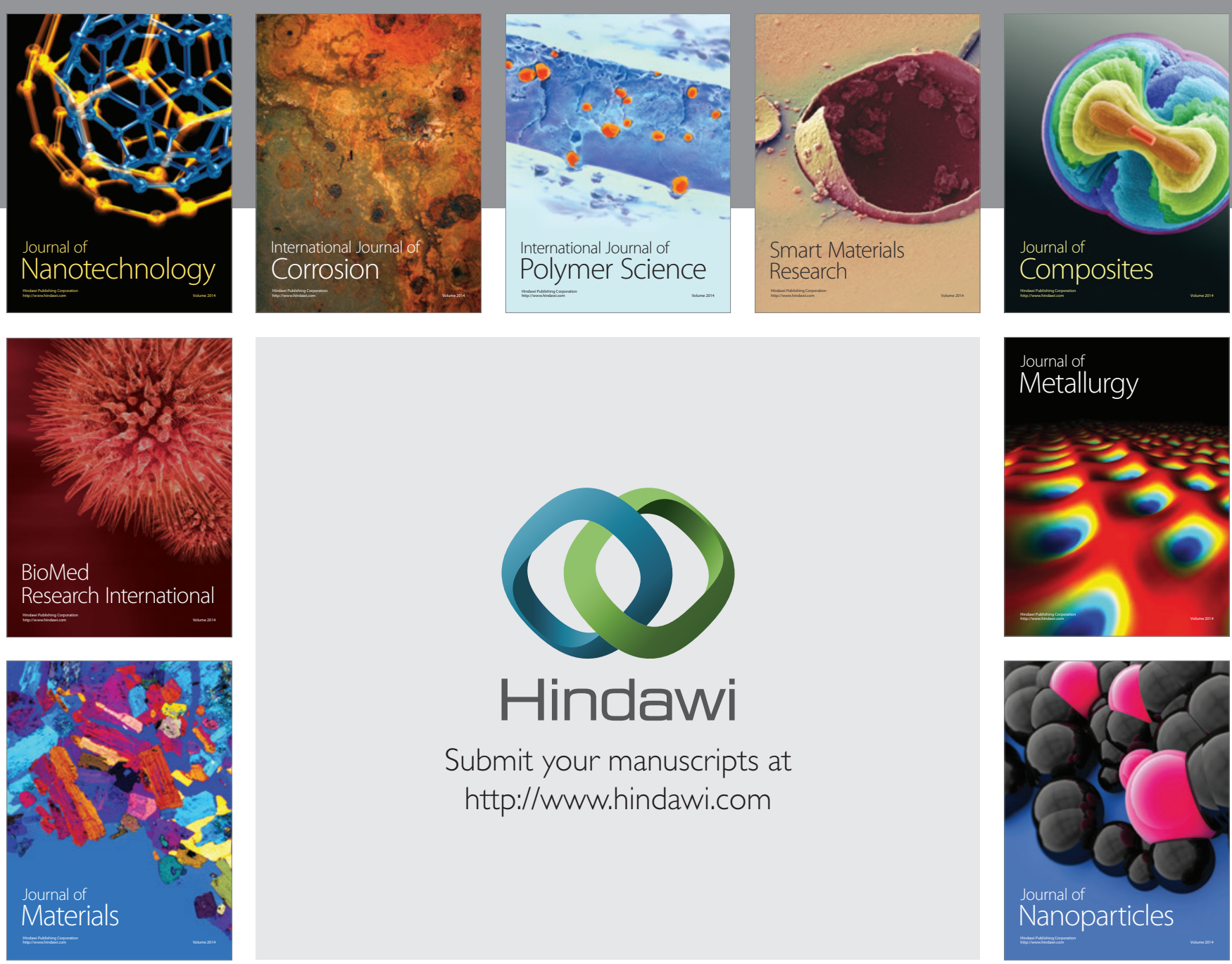

Submit your manuscripts at http://www.hindawi.com
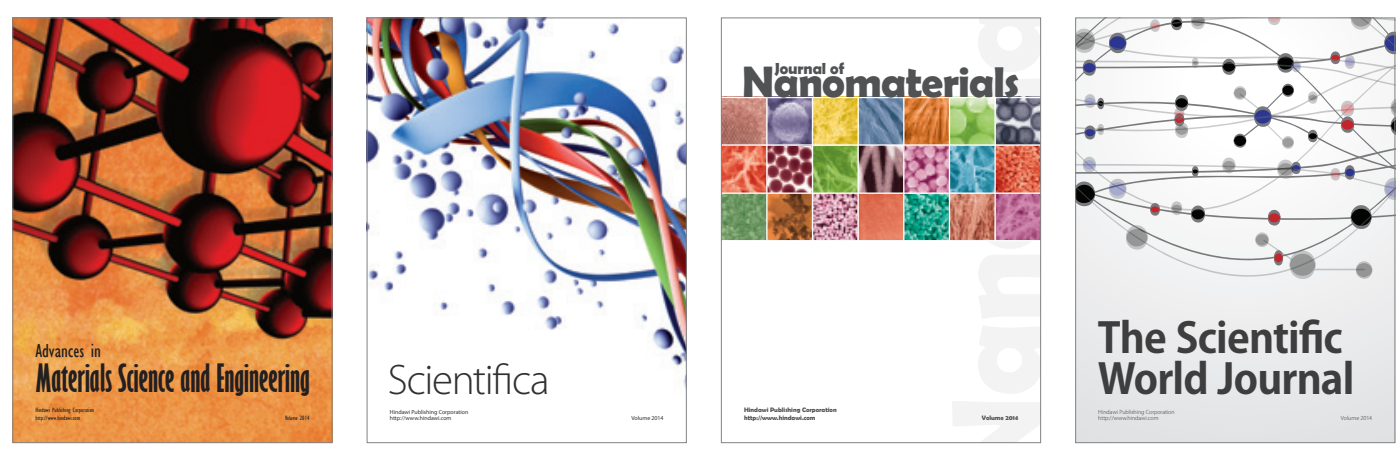

\section{The Scientific World Journal}
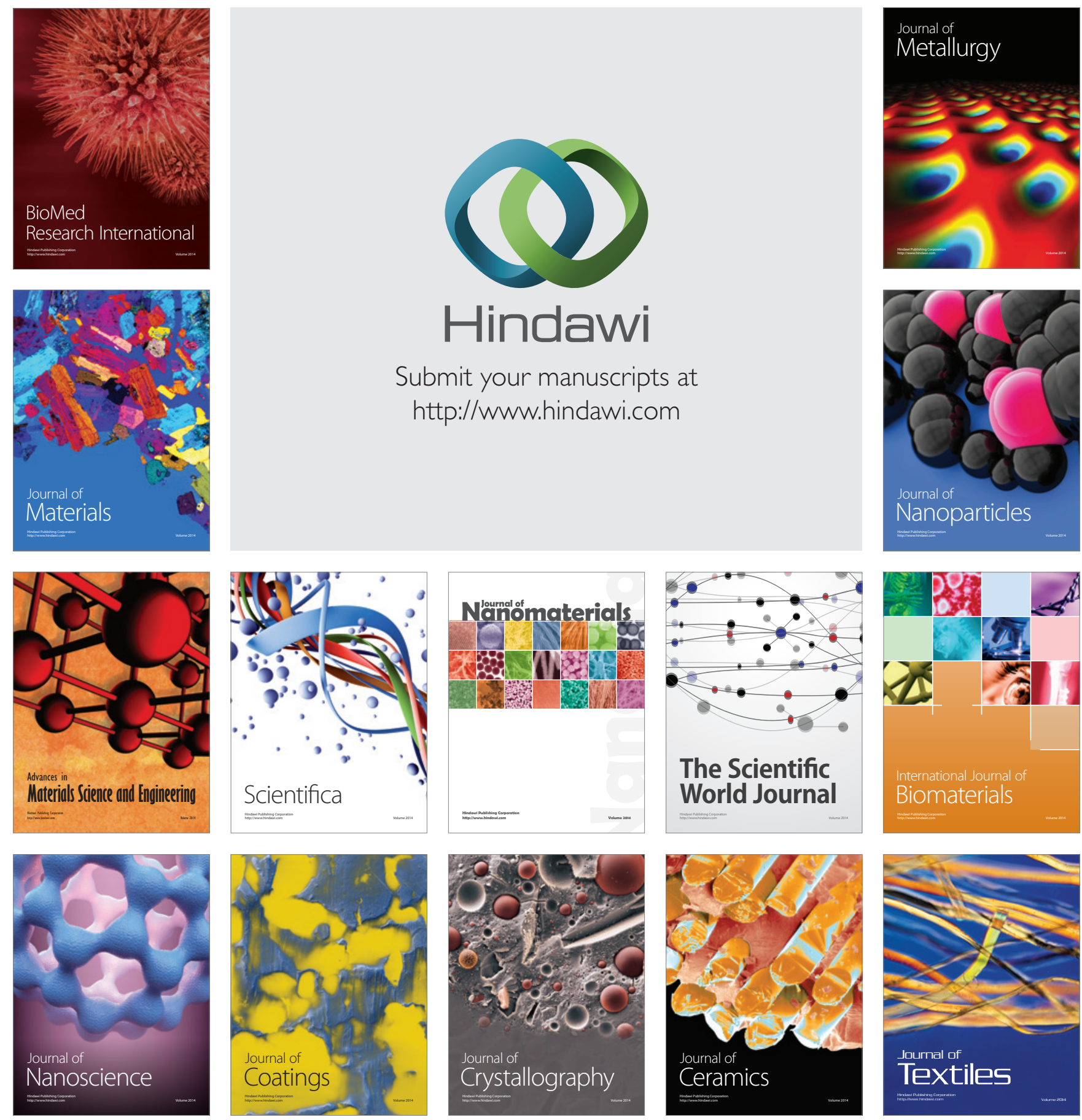